TRANSACTIONS OF THE

AMERICAN MATHEMATICAL SOCIETY

Volume 359, Number 10, October 2007, Pages 4773-4786

S 0002-9947(07)04076-7

Article electronically published on April 24, 2007

\title{
EQUIVALENCE OF DOMAINS ARISING FROM DUALITY OF ORBITS ON FLAG MANIFOLDS III
}

\author{
TOSHIHIKO MATSUKI
}

\begin{abstract}
In Gindikin and Matsuki 2003, we defined a $G_{\mathbb{R}}-K_{\mathbb{C}}$ invariant sub-

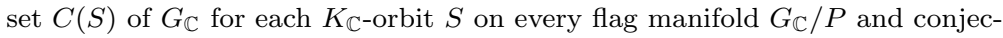
tured that the connected component $C(S)_{0}$ of the identity would be equal to the Akhiezer-Gindikin domain $D$ if $S$ is of nonholomorphic type. This conjecture was proved for closed $S$ in Wolf and Zierau 2000 and 2003, Fels and Huckleberry 2005, and Matsuki 2006 and for open $S$ in Matsuki 2006. It was proved for the other orbits in Matsuki 2006, when $G_{\mathbb{R}}$ is of non-Hermitian type. In this paper, we prove the conjecture for an arbitrary non-closed $K_{\mathbb{C}^{-}}$ orbit when $G_{\mathbb{R}}$ is of Hermitian type. Thus the conjecture is completely solved affirmatively.
\end{abstract}

\section{INTRODUCTION}

Let $G_{\mathbb{C}}$ be a connected complex semisimple Lie group and $G_{\mathbb{R}}$ a connected real form of $G_{\mathbb{C}}$. Let $K_{\mathbb{C}}$ be the complexification in $G_{\mathbb{C}}$ of a maximal compact subgroup $K$ of $G_{\mathbb{R}}$. Let $X=G_{\mathbb{C}} / P$ be a flag manifold of $G_{\mathbb{C}}$, where $P$ is an arbitrary parabolic subgroup of $G_{\mathbb{C}}$. Then there exists a natural one-to-one correspondence between

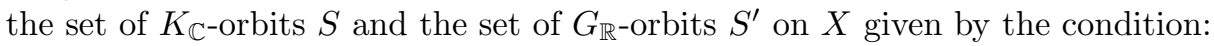

$$
S \leftrightarrow S^{\prime} \Longleftrightarrow S \cap S^{\prime} \text { is non-empty and compact }
$$

(M2]). For each $K_{\mathbb{C}}$-orbit $S$ we defined in [GM1] a subset $C(S)$ of $G_{\mathbb{C}}$ by

$$
C(S)=\left\{x \in G_{\mathbb{C}} \mid x S \cap S^{\prime} \text { is non-empty and compact }\right\}
$$

where $S^{\prime}$ is the $G_{\mathbb{R}}$-orbit on $X$ given by (1.1).

Akhiezer and Gindikin defined a domain $D / K_{\mathbb{C}}$ in $G_{\mathbb{C}} / K_{\mathbb{C}}$ as follows $\left.(\mathrm{AG}]\right)$. Let $\mathfrak{g}_{\mathbb{R}}=\mathfrak{k} \oplus \mathfrak{m}$ denote the Cartan decomposition of $\mathfrak{g}_{\mathbb{R}}=\operatorname{Lie}\left(G_{\mathbb{R}}\right)$ with respect to $K$. Let $\mathfrak{t}$ be a maximal abelian subspace in $i \mathfrak{m}$. Put

$$
\mathfrak{t}^{+}=\left\{Y \in \mathfrak{t}|| \alpha(Y) \mid<\frac{\pi}{2} \text { for all } \alpha \in \Sigma\right\}
$$

where $\Sigma$ is the restricted root system of $\mathfrak{g}_{\mathbb{C}}$ with respect to $\mathfrak{t}$. Then $D$ is defined by

$$
D=G_{\mathbb{R}}\left(\exp \mathfrak{t}^{+}\right) K_{\mathbb{C}}
$$

We conjectured the following in GM1.

Conjecture 1.1 (Conjecture 1.6 in GM1]). Suppose that $X=G_{\mathbb{C}} / P$ is not $K_{\mathbb{C}^{-}}$ homogeneous. Then we will have $C(S)_{0}=D$ for all $K_{\mathbb{C}}$-orbits $S$ of non-holomorphic type on $X$. Here $C(S)_{0}$ is the connected component of $C(S)$ containing the identity.

Received by the editors October 20, 2004 and, in revised form, April 28, 2005.

2000 Mathematics Subject Classification. Primary 14M15, 22E15, 22E46, 32M05.

Key words and phrases. Flag manifolds, symmetric spaces, Stein extensions.

(C)2006 American Mathematical Society Reverts to public domain 28 years from publication 4773 
Remark 1.2. When $G_{\mathbb{R}}$ is of Hermitian type, there exist two special closed $K_{\mathbb{C}^{-}}$ orbits $S_{1}=K_{\mathbb{C}} B / B=Q / B$ and $S_{2}=K_{\mathbb{C}} w_{0} B / B=\bar{Q} w_{0} / B$ on the full flag manifold $G_{\mathbb{C}} / B$, where $Q=K_{\mathbb{C}} B$ is the usual maximal parabolic subgroup of $G_{\mathbb{C}}$ defined by a nontrivial central element in $i \mathfrak{k}$ and $w_{0}$ is the longest element in the Weyl group. For each parabolic subgroup $P$ containing the Borel subgroup $B$, two

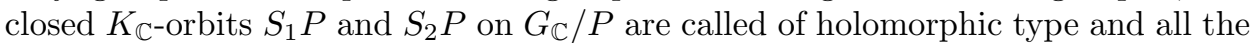

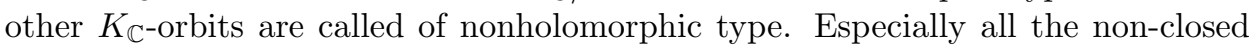

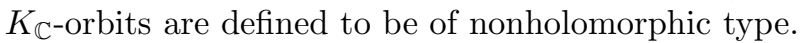

When $G_{\mathbb{R}}$ is of non-Hermitian type, we define that all the $K_{\mathbb{C} \text {-orbits are of }}$ nonholomorphic type.

Let $S_{\text {op }}$ denote the unique open dense $K_{\mathbb{C}^{-}} B$ double coset in $G_{\mathbb{C}}$. Then $S_{\text {op }}^{\prime}$ is the unique closed $G_{\mathbb{R}^{-}} B$ double coset in $G_{\mathbb{C}}$. In this case we see that

$$
C\left(S_{\mathrm{op}}\right)=\left\{x \in G_{\mathbb{C}} \mid x S_{\mathrm{op}} \supset S_{\mathrm{op}}^{\prime}\right\} .
$$

It follows easily that $C\left(S_{\mathrm{op}}\right)$ is a Stein manifold (cf. [GM1, [H]). The connected component $C\left(S_{\mathrm{op}}\right)_{0}$ is often called the Iwasawa domain.

The inclusion

$$
D \subset C\left(S_{\mathrm{op}}\right)_{0}
$$

was proved in $[\mathrm{H}$. (Later $\mathrm{M} 3$ gave a proof without complex analysis.) On the other hand, it was proved in GM1, Proposition 8.1 and Proposition 8.3, that $C\left(S_{\text {op }}\right)_{0} \subset C(S)_{0}$ for all $K_{\mathbb{C}-P}$ double cosets $S$ for any $P$. So we have the inclusion

$$
D \subset C(S)_{0} \text {. }
$$

Hence we have only to prove the converse inclusion

$$
C(S)_{0} \subset D
$$

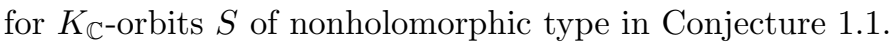

If $S$ is closed in $G_{\mathbb{C}}$, then we can write

$$
C(S)=\left\{x \in G_{\mathbb{C}} \mid x S \subset S^{\prime}\right\} .
$$

So the connected component $C(S)_{0}$ is essentially equal to the cycle space introduced in [WW]. For Hermitian cases the inclusion (1.3) for closed $S$ was proved in WZ2 and WZ3. For non-Hermitian cases it was proved in [FH] and [M4].

When $S$ is the open $K_{\mathbb{C}^{-}} P$ double coset in $G_{\mathbb{C}}$, the inclusion (1.3) was proved in M4 for an arbitrary $P$ generalizing the result in $B$.

Recently the inclusion (1.3) was proved in [M5] for an arbitrary orbit $S$ when $G_{\mathbb{R}}$ is of non-Hermitian type. So the remaining problem was to prove (1.3) for non-closed and non-open orbits when $G_{\mathbb{R}}$ is of Hermitian type.

In this paper we solve this problem.

In the next section we prove the following theorem.

Theorem 1.3. Suppose that $G_{\mathbb{R}}$ is of Hermitian type and let $S$ be a non-closed $K_{\mathbb{C}}-P$ double coset in $G_{\mathbb{C}}$. Then there exist $K_{\mathbb{C}}-B$ double cosets $\widetilde{S}_{1}$ and $\widetilde{S}_{2}$ contained in the boundary $\partial S=S^{c l}-S$ of $S$ such that

$$
x\left(\widetilde{S}_{1} \cup \widetilde{S}_{2}\right)^{c l} \cap S_{0}^{\prime c l} \neq \phi
$$

for all the elements $x$ in the boundary of $D$. Here $S_{0}$ denotes the dense $K_{\mathbb{C}-B}$ double coset in $S$. 
Remark 1.4. It seems that $\widetilde{S_{1}}$ and $\widetilde{S_{2}}$ are always distinct $K_{\mathbb{C}^{-} \text {orbits. But we do }}$ not need this distinctness.

Corollary 1.5. Suppose that $G_{\mathbb{R}}$ is of Hermitian type and let $S$ be a non-closed $K_{\mathbb{C}}-P$ double coset in $G_{\mathbb{C}}$. Then $C(S)_{0}=D$.

Proof. Let $S_{0}$ be as in Theorem 1.3. Let $\Psi$ denote the set of the simple roots in the positive root system for $B$. For each $\alpha \in \Psi$ we define a parabolic subgroup

$$
P_{\alpha}=B \sqcup B w_{\alpha} B
$$

of $G_{\mathbb{C}}$. By GM2, Lemma 2, we can take a sequence $\left\{\alpha_{1}, \ldots, \alpha_{m}\right\}$ of simple roots such that

$$
\operatorname{dim}_{\mathbb{C}} S_{0} P_{\alpha_{1}} \cdots P_{\alpha_{k}}=\operatorname{dim}_{\mathbb{C}} S_{0}+k
$$

for $k=0, \ldots, m=\operatorname{codim}_{\mathbb{C}} S_{0}$. Then it is shown in [M5], Theorem 1.4, that

$$
x \in C(S) \cap D^{c l} \Longrightarrow x S^{c l} \cap S_{\mathrm{op}}^{\prime} P_{\alpha_{m}} \cdots P_{\alpha_{1}}=x S \cap S_{0}^{\prime} .
$$

Let $x$ be an element in the boundary of $D$. Then it follows from Theorem 1.3 that

$$
x(\partial S) \cap S_{0}^{\prime c l} \neq \phi .
$$

If $x$ is also contained in $C(S)$, then it follows from (1.4) that

$$
x(\partial S) \cap S_{\mathrm{op}}^{\prime} P_{\alpha_{m}} \cdots P_{\alpha_{1}}=\phi .
$$

Since $S_{0}^{\prime c l}$ is contained in the closed set $S_{\mathrm{op}}^{\prime} P_{\alpha_{m}} \cdots P_{\alpha_{1}}$, we have

$$
x(\partial S) \cap S_{0}^{\prime c l}=\phi,
$$

a contradiction. Hence $x \notin C(S)$. Thus we have proved $C(S)_{0} \subset D$.

Section 3 is devoted to the explicit computation of the case where $G_{\mathbb{R}}=S p(2, \mathbb{R})$. We use Proposition 3.2 in the proof of Lemma 2.4 in Section 2. Another simple example of the $S U(2,1)$-case is explicitly computed in [M4] Example 1.5.

\section{Proof of Theorem 1.3}

Let $\mathfrak{j}$ be a maximal abelian subspace of $i \mathfrak{k}$. Let $\Delta$ denote the root system of the pair $\left(\mathfrak{g}_{\mathbb{C}}, \mathfrak{j}\right)$. Since $G_{\mathbb{R}}$ is a group of Hermitian type, there exists a nontrivial central element $Z$ of $i \mathfrak{k}$ and we can write

$$
\mathfrak{g}_{\mathbb{C}}=\mathfrak{k}_{\mathbb{C}} \oplus \mathfrak{n} \oplus \overline{\mathfrak{n}}
$$

where $\Delta_{n}^{+}=\{\alpha \in \Delta \mid \alpha(Z)>0\}, \mathfrak{n}=\bigoplus_{\alpha \in \Delta_{n}^{+}} \mathfrak{g}_{\mathbb{C}}(\mathfrak{j}, \alpha)$ and $* \mapsto \bar{*}$ denotes the conjugation in $\mathfrak{g}_{\mathbb{C}}$ with respect to $\mathfrak{g}_{\mathbb{R}}$. Let $Q$ be the maximal parabolic subgroup of $G_{\mathbb{C}}$ defined by $Q=K_{\mathbb{C}} \exp \mathfrak{n}$. Let $\Delta^{+}$be a positive system of $\Delta$ containing $\Delta_{n}^{+}$. Then it defines a Borel subgroup $B=B\left(\mathfrak{j}, \Delta^{+}\right)$of $G_{\mathbb{C}}$ contained in $Q$.

Let $P$ be a parabolic subgroup of $G_{\mathbb{C}}$ containing $B$. Let $S$ be a non-closed $K_{\mathbb{C}-} P$ double coset in $G_{\mathbb{C}}$ and let $S_{0}$ denote the dense $K_{\mathbb{C}^{-}} B$ double coset in $S$. By [M1, Theorem 2, we can write

$$
S_{0}=K_{\mathbb{C}} c_{\gamma_{1}} \cdots c_{\gamma_{k}} w B
$$

with some $w \in W$ and a strongly orthogonal system $\left\{\gamma_{1}, \ldots, \gamma_{k}\right\}$ of roots in $\Delta_{n}^{+}$. Here $W$ is the Weyl group of $\Delta$ and

$$
c_{\gamma_{j}}=\exp (X-\bar{X})
$$

with some $X \in \mathfrak{g}_{\mathbb{C}}\left(\mathfrak{j}, \gamma_{j}\right)$ such that $c_{\gamma_{j}}^{2}$ is the reflection with respect to $\gamma_{j}$. 
Let $\Theta$ denote the subset of $\Psi$ such that $P=B W_{\Theta} B$ where $W_{\Theta}$ is the subgroup of $W$ generated by $\left\{w_{\alpha} \mid \alpha \in \Theta\right\}$. Let $\Delta_{\Theta}$ denote the subset of $\Delta$ defined by

$$
\Delta_{\Theta}=\left\{\beta \in \Delta \mid \beta=\sum_{\alpha \in \Theta} n_{\alpha} \alpha \text { for some } n_{\alpha} \in \mathbb{Z}\right\} .
$$

If $\gamma_{j} \in w \Delta_{\Theta}$ for all $j=1, \ldots, k$, then it follows that $c_{\gamma_{j}} \in w P w^{-1}$ for all $j=1, \ldots, k$ and therefore

$$
S w^{-1}=S_{0} P w^{-1}=K_{\mathbb{C}} c_{\gamma_{1}} \cdots c_{\gamma_{k}} w P w^{-1}=K_{\mathbb{C}} w P w^{-1}
$$

becomes closed in $G_{\mathbb{C}}$, contradicting the assumption. Hence there exists a $j$ such that $\gamma_{j} \notin w \Delta_{\Theta}$. Replacing the order of $\gamma_{1}, \ldots, \gamma_{k}$, we may assume that

$$
\gamma_{1} \notin w \Delta_{\Theta} .
$$

Let $\mathfrak{l}$ denote the complex Lie subalgebra of $\mathfrak{g}_{\mathbb{C}}$ generated by $\mathfrak{g}_{\mathbb{C}}\left(\mathfrak{j}, \gamma_{1}\right) \oplus \mathfrak{g}_{\mathbb{C}}\left(\mathfrak{j},-\gamma_{1}\right)$ which is isomorphic to $\mathfrak{s l}(2, \mathbb{C})$ and let $L$ be the analytic subgroup of $G_{\mathbb{C}}$ for ll. Then we have $\left(L \cap K_{\mathbb{C}}\right) c_{\gamma_{1}}\left(L \cap w B w^{-1}\right)=\left(L \cap K_{\mathbb{C}}\right) c_{\gamma_{1}}^{-1}\left(L \cap w B w^{-1}\right)$ since both of the double cosets are open dense in $L$. Hence we have

$$
S_{0}=K_{\mathbb{C}} c_{\gamma_{1}} \cdots c_{\gamma_{k}} w B=K_{\mathbb{C}} c_{\gamma_{1}}^{-1} c_{\gamma_{2}} \cdots c_{\gamma_{k}} w B=K_{\mathbb{C}} c_{\gamma_{1}} \cdots c_{\gamma_{k}} w_{\gamma_{1}} w B .
$$

If $\gamma_{1} \notin w \Delta^{+}$, then $\gamma_{1} \in w_{\gamma_{1}} w \Delta^{+}$. So we may assume

$$
\gamma_{1} \in w \Delta^{+}
$$

replacing $w$ with $w_{\gamma_{1}} w$ if necessary. Let $\ell$ denote the real rank of $G_{\mathbb{R}}$.

Lemma 2.1. There exists a maximal strongly orthgonal system $\left\{\beta_{1}, \ldots, \beta_{\ell}\right\}$ of roots in $\Delta_{n}^{+}$satisfying the following conditions:

(i) If $\gamma_{1}$ is a long root of $\Delta$, then $\beta_{1}=\gamma_{1}$ and $\gamma_{2}, \ldots, \gamma_{k} \in \mathbb{R} \beta_{2} \oplus \cdots \oplus \mathbb{R} \beta_{\ell}$. (If the roots in $\Delta$ have the same length, then we define that all the roots are long roots.)

(ii) If $\gamma_{1}$ is a short root of $\Delta$, then $\gamma_{1} \in \mathbb{R} \beta_{1} \oplus \mathbb{R} \beta_{2}$ and $\gamma_{2}, \ldots, \gamma_{k} \in \mathbb{R} \beta_{3} \oplus \cdots \oplus$ $\mathbb{R} \beta_{\ell}$.

Proof. First suppose that $\mathfrak{g}_{\mathbb{R}}$ is of type AIII, DIII, EIII, EVII or DI (of real rank $2)$. Then the roots in $\Delta$ have the same length. So we have only to take $\beta_{j}=\gamma_{j}$ for $j=1, \ldots, k$ and choose an orthogonal system $\left\{\beta_{1}, \ldots, \beta_{\ell}\right\}$ of roots in $\Delta_{n}^{+}$containing $\left\{\beta_{1}, \ldots, \beta_{k}\right\}$.

Next suppose that $\mathfrak{g}_{\mathbb{R}} \cong \mathfrak{s p}(\ell, \mathbb{R})$. Write

$$
\Delta=\left\{ \pm e_{r} \pm e_{s} \mid 1 \leq r<s \leq \ell\right\} \sqcup\left\{ \pm 2 e_{r} \mid 1 \leq r \leq \ell\right\}
$$

and

$$
\Delta_{n}^{+}=\left\{e_{r}+e_{s} \mid 1 \leq r<s \leq \ell\right\} \sqcup\left\{2 e_{r} \mid 1 \leq r \leq \ell\right\}
$$

as usual using an orthonormal basis $\left\{e_{1}, \ldots, e_{\ell}\right\}$ of $\boldsymbol{j}^{*}$. If $\gamma_{1}=2 e_{r}$, then $\left\{\beta_{2}, \ldots, \beta_{\ell}\right\}$ $=\left\{2 e_{s} \mid s \neq r\right\}$ satisfies condition (i). If $\gamma_{1}=e_{r}+e_{s}$ with $r \neq s$, then we put $\beta_{1}=$ $2 e_{r}$ and $\beta_{2}=2 e_{s}$. Assertion (ii) is clear if we put $\left\{\beta_{3}, \ldots, \beta_{\ell}\right\}=\left\{2 e_{p} \mid p \neq r, s\right\}$.

Finally suppose that $\mathfrak{g}_{\mathbb{R}}=\mathfrak{s o}(2,2 p-1)$ with $p \geq 2$. Then the real rank of $\mathfrak{g}_{\mathbb{R}}$ is two, and we can write

$$
\Delta=\left\{ \pm e_{r} \pm e_{s} \mid 1 \leq r<s \leq p\right\} \sqcup\left\{ \pm e_{r} \mid 1 \leq r \leq p\right\}
$$

and

$$
\Delta_{n}^{+}=\left\{e_{1} \pm e_{s} \mid 2 \leq s \leq p\right\} \sqcup\left\{e_{1}\right\}
$$


with an orthonormal basis $\left\{e_{1}, \ldots, e_{p}\right\}$ of $\mathfrak{j}^{*}$. If $k=2$, then we have $\gamma_{1}=\beta_{1}=e_{1} \pm e_{s}$ and $\gamma_{2}=\beta_{2}=e_{1} \mp e_{s}$ with some $s$. If $k=1$ and $\gamma_{1}=e_{1} \pm e_{s}$, then $\beta_{1}=\gamma_{1}$ and $\beta_{2}=e_{1} \mp e_{s}$. If $k=1$ and $\gamma_{1}=e_{1}$, then we may put $\beta_{1}=e_{1}+e_{2}$ and $\beta_{2}=e_{1}-e_{2}$.

Definition 2.2. (i) Define a subroot system $\Delta_{1}$ of $\Delta$ as follows.

If $\gamma_{1}$ is a long root of $\Delta$, then we put

$$
\Delta_{1}=\left\{ \pm \beta_{1}\right\}=\left\{ \pm \gamma_{1}\right\} .
$$

On the other hand if $\gamma_{1}$ is a short root of $\Delta$, then we put

$$
\Delta_{1}=\Delta \cap\left(\mathbb{R} \beta_{1} \oplus \mathbb{R} \beta_{2}\right)
$$

(which is of type $\mathrm{C}_{2}$ ).

(ii) Put $\Delta_{2}=\left\{\alpha \in \Delta \mid \alpha\right.$ is orthogonal to $\left.\Delta_{1}\right\}$.

(iii) Let $\mathfrak{l}_{j}$ denote the complex Lie subalgebras of $\mathfrak{g}_{\mathbb{C}}$ generated by $\bigoplus_{\alpha \in \Delta_{j}} \mathfrak{g}_{\mathbb{C}}(\mathfrak{j}, \alpha)$ for $j=1,2$.

(iv) Let $L_{1}$ and $L_{2}$ denote the analytic subgroups of $G_{\mathbb{C}}$ for $\mathfrak{l}_{1}$ and $\mathfrak{l}_{2}$, respectively.

It follows from Lemma 2.1 that

$$
c_{\gamma_{1}} \in L_{1} \quad \text { and that } \quad c_{\gamma_{2}} \cdots c_{\gamma_{k}} \in L_{2} .
$$

Let $X_{j}$ be nonzero root vectors in $\mathfrak{g}_{\mathbb{C}}\left(\mathfrak{j}, \beta_{j}\right)$ for $j=1, \ldots, \ell$. Then we can define a maximal abelian subspace

$$
\mathfrak{t}=\mathbb{R}\left(X_{1}-\overline{X_{1}}\right) \oplus \cdots \oplus \mathbb{R}\left(X_{\ell}-\overline{X_{\ell}}\right)
$$

in $i \mathfrak{m}$ and a maximal abelian subspace

$$
\mathfrak{a}=\mathbb{R}\left(X_{1}+\overline{X_{1}}\right) \oplus \cdots \oplus \mathbb{R}\left(X_{\ell}+\overline{X_{\ell}}\right)
$$

in $\mathfrak{m}$ as in GM1, Section 2. Since the restricted root system $\Sigma(\mathfrak{t})$ is of type $\mathrm{BC}_{\ell}$ or $\mathrm{C}_{\ell}$, the set $\mathfrak{t}^{+}$is defined by the long roots in $\Sigma(\mathfrak{t})$. Hence it is of the form

$$
\mathfrak{t}^{+}=\left\{Y_{1}+\cdots+Y_{\ell} \mid Y_{j} \in \mathfrak{t}_{j}^{+}\right\}
$$

where $\mathfrak{t}_{j}^{+}=\left\{s\left(X_{j}-\overline{X_{j}}\right) \mid-(\pi / 4)<s<\pi / 4\right\}$ by a suitable normalization of $X_{j}$ for $j=1, \ldots, \ell$.

Put $T^{+}=\exp ^{+}$and $A=\exp \mathfrak{a}$. Then it is shown in [GM1, Lemma 2.1, that $A Q=T^{+} Q$ and hence that

$$
G_{\mathbb{R}} Q=K A Q=K T^{+} Q
$$

by the Cartan decomposition $G_{\mathbb{R}}=K A K$. The closure of $G_{\mathbb{R}} Q$ in $G_{\mathbb{C}}$ is written as

$$
\left(G_{\mathbb{R}} Q\right)^{c l}=G_{\mathbb{R}} Q \sqcup G_{\mathbb{R}} c_{\beta_{1}} Q \sqcup G_{\mathbb{R}} c_{\beta_{1}} c_{\beta_{2}} Q \sqcup \cdots \sqcup G_{\mathbb{R}} c_{\beta_{1}} \cdots c_{\beta_{\ell}} Q
$$

where $c_{\beta_{j}}=\exp (\pi / 4)\left(X_{j}-\overline{X_{j}}\right)$ for $j=1, \ldots, \ell$ ([WZ1], Theorem 3.8). We also see that

$$
G_{\mathbb{R}} c_{\beta_{1}} \cdots c_{\beta_{k}} Q=K c_{\beta_{1}} \cdots c_{\beta_{k}} T_{k+1}^{+} \cdots T_{\ell}^{+} Q
$$

where $T_{j}^{+}=\exp \mathfrak{t}_{j}^{+}$since we can consider the action of the Weyl group $W_{K}(T)$ on $T$ which is of type $\mathrm{BC}_{\ell}$.

By the map

$$
\iota: x K_{\mathbb{C}} \mapsto(x Q, x \bar{Q})
$$


the complex symmetric space $G_{\mathbb{C}} / K_{\mathbb{C}}$ is embedded in $G_{\mathbb{C}} / Q \times G_{\mathbb{C}} / \bar{Q}$ (WZ2]). It is shown in [BHH], Section 3, and [GM1, Proposition 2.2, that

$$
\iota\left(D / K_{\mathbb{C}}\right)=G_{\mathbb{R}} Q / Q \times G_{\mathbb{R}} \bar{Q} / \bar{Q} .
$$

Lemma 2.3. Suppose that

$$
\iota\left(x K_{\mathbb{C}}\right) \in G_{\mathbb{R}} c_{\beta_{1}} Q / Q \times G_{\mathbb{R}} \bar{Q} / \bar{Q}
$$

and that $\gamma_{1}$ is a long root of $\Delta_{n}^{+}$. (If the roots in $\Delta$ have the same length, then we define that all the roots are long roots.) Define a $K_{\mathbb{C}-B}$ double coset $\widetilde{S}_{1}$ by

$$
\widetilde{S}_{1}=K_{\mathbb{C}} c_{\gamma_{2}} \cdots c_{\gamma_{k}} w B
$$

Then $\widetilde{S}_{1}$ is contained in $\partial S=S^{c l}-S$ and

$$
x \widetilde{S}_{1} \cap S_{0}^{\prime} \neq \phi .
$$

Proof. It is clear that we may replace $x$ by any elements in the double coset $G_{\mathbb{R}} x K_{\mathbb{C}}$. By the left $G_{\mathbb{R}}$-action we may assume that $x \in \bar{Q}$. By the right $K_{\mathbb{C} \text {-action we may }}$ moreover assume that $x \in \bar{N}$ since $\bar{Q}=\bar{N} K_{\mathbb{C}}$. Since $K=K_{\mathbb{C}} \cap G_{\mathbb{R}}$ normalizes $\bar{N}$, we may assume by (2.1) that

$$
x Q=c_{\beta_{1}} t_{2} \cdots t_{\ell} Q
$$

with some $t_{j} \in T_{j}^{+}$for $j=2, \ldots, \ell$. As in [WZ2, we write

$$
c_{\beta_{1}}=c_{\gamma_{1}}=c=c^{-} c^{+} \text {and } t_{j}=t_{j}^{-} t_{j}^{+} \text {for } j=2, \ldots, \ell
$$

with $c^{-}, t_{j}^{-} \in \bar{N}$ and $c^{+}, t_{j}^{+} \in Q$. Then we have

$$
x=c^{-} t_{2}^{-} \cdots t_{\ell}^{-} \text {. }
$$

It follows from Lemma 2.1 and Definition 2.2 that $c_{\gamma_{2}} \cdots c_{\gamma_{k}} \in L_{2}$. Since $\operatorname{Ad}\left(c_{\gamma_{2}} \cdots c_{\gamma_{k}}\right) j$ is $\theta$-stable, the double cosets

$$
S_{L_{2}}=\left(L_{2} \cap K_{\mathbb{C}}\right) c_{\gamma_{2}} \cdots c_{\gamma_{k}}\left(L_{2} \cap w B w^{-1}\right)
$$

and

$$
S_{L_{2}}^{\prime}=\left(L_{2} \cap G_{\mathbb{R}}\right) c_{\gamma_{2}} \cdots c_{\gamma_{k}}\left(L_{2} \cap w B w^{-1}\right)
$$

correspond by the duality ( M1], Theorem 2 ).

It follows from Lemma 2.1 (i) and Definition 2.2 that

$$
c^{ \pm} \in L_{1} \quad \text { and } \quad t_{2}^{ \pm}, \ldots, t_{\ell}^{ \pm} \in L_{2} .
$$

It follows moreover from Definition 2.2 (i) that $\mathfrak{l}_{1} \cong \mathfrak{s l}(2, \mathbb{C})$.

Write $y=t_{2}^{-} \cdots t_{\ell}^{-}$. Then we have

$$
y Q=t_{2} \cdots t_{\ell} Q \subset T^{+} Q \subset G_{\mathbb{R}} Q
$$

and

$$
y \bar{Q}=\bar{Q} \subset G_{\mathbb{R}} \bar{Q}
$$

Hence we have

$$
y \in L_{2} \cap\left(C\left(S_{1}\right) \cap C\left(S_{2}\right)\right)=L_{2} \cap D
$$

by GM1], (1.3). By the inclusion (1.2) this implies that the set $y S_{L_{2}} \cap S_{L_{2}}^{\prime}$ is nonempty and closed in $L_{2}$. Take an element $z$ of $y S_{L_{2}} \cap S_{L_{2}}^{\prime}$. 
Since $\gamma_{1} \in w \Delta^{+}$, we have $c^{+} \in w B w^{-1}$. Since $c^{+} \in L_{1}$ commutes with elements in $L_{2}$, we have

$$
\begin{aligned}
c z \in c y S_{L_{2}} & =c^{-} c^{+} y\left(L_{2} \cap K_{\mathbb{C}}\right) c_{\gamma_{2}} \cdots c_{\gamma_{k}}\left(L_{2} \cap w B w^{-1}\right) \\
& =c^{-} y\left(L_{2} \cap K_{\mathbb{C}}\right) c_{\gamma_{2}} \cdots c_{\gamma_{k}} c^{+}\left(L_{2} \cap w B w^{-1}\right) \\
& \subset c^{-} y K_{\mathbb{C}} c_{\gamma_{2}} \cdots c_{\gamma_{k}} w B w^{-1}=x \widetilde{S}_{1} w^{-1} .
\end{aligned}
$$

On the other hand we have

$$
\begin{aligned}
c z \in c S_{L_{2}}^{\prime} & =c\left(L_{2} \cap G_{\mathbb{R}}\right) c_{\gamma_{2}} \cdots c_{\gamma_{k}}\left(L_{2} \cap w B w^{-1}\right) \\
& =\left(L_{2} \cap G_{\mathbb{R}}\right) c_{\gamma_{1}} c_{\gamma_{2}} \cdots c_{\gamma_{k}}\left(L_{2} \cap w B w^{-1}\right) \subset S_{0}^{\prime} w^{-1} .
\end{aligned}
$$

Hence $x \widetilde{S}_{1} \cap S_{0}^{\prime} \neq \phi$. It is clear that $\widetilde{S}_{1} \subset S_{0}^{c l}=S^{c l}$ because

$$
\left(L_{1} \cap K_{\mathbb{C}}\right)\left(L_{1} \cap w B w^{-1}\right) \subset\left(\left(L_{1} \cap K_{\mathbb{C}}\right) c\left(L_{1} \cap w B w^{-1}\right)\right)^{c l}=L_{1} .
$$

Now we will prove $\widetilde{S}_{1} \not \subset S$. Consider the map

$$
\varphi: K_{\mathbb{C}} \backslash G_{\mathbb{C}} / B \ni K_{\mathbb{C}} g B \mapsto B \theta(g)^{-1} g B \in B \backslash G_{\mathbb{C}} / B
$$

introduced in $\left[\mathrm{Sp}\right.$, where $\theta$ is the holomorphic involution in $G_{\mathbb{C}}$ defining $K_{\mathbb{C}}$. We have

and

$$
\varphi\left(\widetilde{S}_{1}\right)=B w^{-1} w_{\gamma_{2}} \cdots w_{\gamma_{k}} w B
$$

$$
\varphi(S)=\varphi\left(S_{0} P\right) \subset P w^{-1} w_{\gamma_{1}} \cdots w_{\gamma_{k}} w P=B W_{\Theta} w^{-1} w_{\gamma_{1}} \cdots w_{\gamma_{k}} w W_{\Theta} B .
$$

So we have only to show

$$
w^{-1} w_{\gamma_{2}} \cdots w_{\gamma_{k}} w \notin W_{\Theta} w^{-1} w_{\gamma_{1}} \cdots w_{\gamma_{k}} w W_{\Theta} .
$$

Let $Z$ be an element in $\mathfrak{j}$ defining $P$. This implies that $Z$ is dominant for $\Delta^{+}$and that $\{\alpha \in \Psi \mid \alpha(Z)=0\}=\Theta$. Let $w_{1}$ and $w_{2}$ be elements in $W_{\Theta}$. Let $B($, denote the Killing form on $\mathfrak{g}$ and let $Y_{\gamma_{1}}$ denote the element in $\mathfrak{j}$ such that

$$
\gamma_{1}(Y)=B\left(Y, Y_{\gamma_{1}}\right) \text { for all } Y \in \mathfrak{j}
$$

Then we have

$$
\begin{aligned}
& B\left(Z, w^{-1} w_{\gamma_{2}} \cdots w_{\gamma_{k}} w Z\right)-B\left(Z, w_{1} w^{-1} w_{\gamma_{1}} w_{\gamma_{2}} \cdots w_{\gamma_{k}} w w_{2} Z\right) \\
= & B\left(w Z-w_{\gamma_{1}} w Z, w_{\gamma_{2}} \cdots w_{\gamma_{k}} w Z\right) \\
= & \frac{2 B\left(Y_{\gamma_{1}}, w Z\right)}{B\left(Y_{\gamma_{1}}, Y_{\gamma_{1}}\right)} B\left(Y_{\gamma_{1}}, w_{\gamma_{2}} \cdots w_{\gamma_{k}} w Z\right) \\
= & \frac{2 B\left(Y_{\gamma_{1}}, w Z\right)^{2}}{B\left(Y_{\gamma_{1}}, Y_{\gamma_{1}}\right)}>0
\end{aligned}
$$

since $\gamma_{1} \notin w \Delta_{\Theta}$. Thus we have proved (2.2).

Lemma 2.4. Suppose that

$$
\iota\left(x K_{\mathbb{C}}\right) \in G_{\mathbb{R}} c_{\beta_{1}} Q / Q \times G_{\mathbb{R}} \bar{Q} / \bar{Q}
$$

and that $\gamma_{1}$ is a short root of $\Delta_{n}^{+}$. (We assume that $\mathfrak{g}_{\mathbb{R}} \cong \mathfrak{s p}(\ell, \mathbb{R})$ or $\mathfrak{s o}(2,2 p-1)$ with $p \geq 2$.) Define a $K_{\mathbb{C}}-B$ double coset $\widetilde{S}_{1}$ by $\widetilde{S}_{1}=K_{\mathbb{C}} g c_{\gamma_{2}} \cdots c_{\gamma_{k}} w B$ where

$$
g= \begin{cases}e & \text { if } \gamma_{1} \text { is the simple short root of } \Delta_{1}^{+}, \\ c_{\beta} & \text { if } \gamma_{1} \text { is the non-simple short root of } \Delta_{1}^{+} .\end{cases}
$$


Here $\Delta_{1}^{+}=\Delta_{1} \cap w \Delta^{+}$and $\beta$ is the long simple root of $\Delta_{1}^{+}$. Then $\widetilde{S}_{1}$ is contained in $\partial S=S^{c l}-S$ and

$$
x \widetilde{S}_{1} \cap S_{0}^{\prime c l} \neq \phi .
$$

Proof. It follows from Lemma 2.1 (ii) and Definition 2.2 that

$$
c_{\beta_{1}}^{ \pm}, t_{2}^{ \pm} \in L_{1} \quad \text { and } \quad t_{3}^{ \pm}, \ldots, t_{\ell}^{ \pm} \in L_{2} .
$$

It follows moreover from Definition 2.2 (i) that $\mathfrak{l}_{1} \cong \mathfrak{s p}(2, \mathbb{C})$.

Write $y=t_{3}^{-} \cdots t_{\ell}^{-}$. Then by the same argument as in the proof of Lemma 2.3 we see that the set $y S_{L_{2}} \cap S_{L_{2}}^{\prime}$ is nonempty and closed in $L_{2}$. Take an element $z$ of $y S_{L_{2}} \cap S_{L_{2}}^{\prime}$.

The positive system $\Delta_{1}^{+}$of $\Delta_{1}$ consists of two long roots and two short roots. Since $\gamma_{1} \in \Delta_{1}^{+}, \gamma_{1}$ is either of these two short roots. Write $x_{1}=c_{\beta_{1}}^{-} t_{2}^{-}$.

First assume that $\gamma_{1}$ is the simple short root of $\Delta_{1}^{+}$. Then it follows from Proposition 3.2 (i) in the next section that

$$
x_{1}\left(L_{1} \cap K_{\mathbb{C}}\right)\left(L_{1} \cap w B w^{-1}\right) \cap\left(\left(L_{1} \cap G_{\mathbb{R}}\right) c_{\gamma_{1}}\left(L_{1} \cap w B w^{-1}\right)\right)^{c l}
$$

is nonempty. Note that $L_{1} \cap w B w^{-1}$ and $\gamma_{1}$ correspond to $w_{\beta_{2}} B w_{\beta_{2}}^{-1}$ and $\delta$ in the next section, respectively. Let $z_{1}$ be an element of (2.3). Then we have

$$
\begin{aligned}
z_{1} z & \in x_{1}\left(L_{1} \cap K_{\mathbb{C}}\right)\left(L_{1} \cap w B w^{-1}\right) y S_{L_{2}} \\
& =x_{1}\left(L_{1} \cap K_{\mathbb{C}}\right)\left(L_{1} \cap w B w^{-1}\right) y\left(L_{2} \cap K_{\mathbb{C}}\right) c_{\gamma_{2}} \cdots c_{\gamma_{k}}\left(L_{2} \cap w B w^{-1}\right) \\
& =x_{1} y\left(L_{1} \cap K_{\mathbb{C}}\right)\left(L_{2} \cap K_{\mathbb{C}}\right) c_{\gamma_{2}} \cdots c_{\gamma_{k}}\left(L_{1} \cap w B w^{-1}\right)\left(L_{2} \cap w B w^{-1}\right) \\
& \subset x K_{\mathbb{C}} c_{\gamma_{2}} \cdots c_{\gamma_{k}} w B w^{-1}=x \widetilde{S}_{1} w^{-1}
\end{aligned}
$$

and

$$
\begin{aligned}
z_{1} z & \in\left(\left(L_{1} \cap G_{\mathbb{R}}\right) c_{\gamma_{1}}\left(L_{1} \cap w B w^{-1}\right)\right)^{c l} S_{L_{2}}^{\prime} \\
& =\left(\left(L_{1} \cap G_{\mathbb{R}}\right) c_{\gamma_{1}}\left(L_{1} \cap w B w^{-1}\right)\right)^{c l}\left(L_{2} \cap G_{\mathbb{R}}\right) c_{\gamma_{2}} \cdots c_{\gamma_{k}}\left(L_{2} \cap w B w^{-1}\right) \\
& \subset\left(G_{\mathbb{R}} c_{\gamma_{1}} c_{\gamma_{2}} \cdots c_{\gamma_{k}} w B w^{-1}\right)^{c l}={S_{0}^{\prime}}^{c l} w^{-1} .
\end{aligned}
$$

So we have $x \widetilde{S}_{1} \cap{S_{0}^{\prime}}^{c l} \neq \phi$. We can prove $\widetilde{S}_{1} \subset S^{c l}-S$ by the same arguments as in the proof of Lemma 2.3.

Next assume that $\gamma_{1}$ is the non-simple short root of $\Delta_{1}^{+}$. Then it follows from Proposition 3.2 (ii) in the next section that

$$
x_{1}\left(L_{1} \cap K_{\mathbb{C}}\right) c_{\beta}\left(L_{1} \cap w B w^{-1}\right) \cap\left(\left(L_{1} \cap G_{\mathbb{R}}\right) c_{\gamma_{1}}\left(L_{1} \cap w B w^{-1}\right)\right)^{c l}
$$

is nonempty. Note that $L_{1} \cap w B w^{-1}, \gamma_{1}$ and $\beta$ correspond to $B, \delta$ and $\beta_{2}$ in the next section, respectively. By the same argument as above we can prove

$$
x \widetilde{S}_{1} \cap S_{0}^{\prime c l} \neq \phi .
$$

It follows from Remark 3.3 that $\widetilde{S}_{1} \subset S^{c l}$. Finally we will prove that $\widetilde{S}_{1} \not \subset S$. Using the same argument as in the proof of Lemma 2.3, we have only to show

$$
w^{-1} w_{\beta} w_{\gamma_{2}} \cdots w_{\gamma_{k}} w \notin W_{\Theta} w^{-1} w_{\gamma_{1}} \cdots w_{\gamma_{k}} w W_{\Theta}
$$

Let $Z$ and $Y_{\gamma_{1}}$ be as in the proof of Lemma 2.3. Define $Y_{\beta} \in \mathfrak{j}$ so that

$$
\beta(Y)=B\left(Y, Y_{\beta}\right) \quad \text { for all } Y \in \mathfrak{j} .
$$


Then we have

$$
\begin{aligned}
& B\left(Z, w^{-1} w_{\beta} w_{\gamma_{2}} \cdots w_{\gamma_{k}} w Z\right)-B\left(Z, w_{1} w^{-1} w_{\gamma_{1}} w_{\gamma_{2}} \cdots w_{\gamma_{k}} w w_{2} Z\right) \\
= & B\left(w_{\beta} w Z-w_{\gamma_{1}} w Z, w_{\gamma_{2}} \cdots w_{\gamma_{k}} w Z\right) \\
= & B\left(w Z-w_{\gamma_{1}} w Z, w_{\gamma_{2}} \cdots w_{\gamma_{k}} w Z\right)-B\left(w Z-w_{\beta} w Z, w_{\gamma_{2}} \cdots w_{\gamma_{k}} w Z\right) \\
= & \frac{2 B\left(Y_{\gamma_{1}}, w Z\right)}{B\left(Y_{\gamma_{1}}, Y_{\gamma_{1}}\right)} B\left(Y_{\gamma_{1}}, w_{\gamma_{2}} \cdots w_{\gamma_{k}} w Z\right)-\frac{2 B\left(Y_{\beta}, w Z\right)}{B\left(Y_{\beta}, Y_{\beta}\right)} B\left(Y_{\beta}, w_{\gamma_{2}} \cdots w_{\gamma_{k}} w Z\right) \\
= & \frac{2 B\left(Y_{\gamma_{1}}, w Z\right)^{2}}{B\left(Y_{\gamma_{1}}, Y_{\gamma_{1}}\right)}-\frac{2 B\left(Y_{\beta}, w Z\right)^{2}}{B\left(Y_{\beta}, Y_{\beta}\right)}>0
\end{aligned}
$$

for $w_{1}, w_{2} \in W_{\Theta}$ since

$B\left(Y_{\gamma_{1}}, w Z\right)>0, \quad 0 \leq B\left(Y_{\beta}, w Z\right) \leq B\left(Y_{\gamma_{1}}, w Z\right) \quad$ and $\quad B\left(Y_{\beta}, Y_{\beta}\right)=2 B\left(Y_{\gamma_{1}}, Y_{\gamma_{1}}\right)$.

Thus we have proved (2.4).

Using the conjugation on $G_{\mathbb{C}}$ with respect to the real form $G_{\mathbb{R}}$, the following follows from Lemma 2.3 and Lemma 2.4.

Corollary 2.5. Suppose that

$$
\iota\left(x K_{\mathbb{C}}\right) \in G_{\mathbb{R}} Q / Q \times G_{\mathbb{R}} \overline{c_{\beta_{1}}} \bar{Q} / \bar{Q} .
$$

Then there exists a $K_{\mathbb{C}}-B$ double coset $\widetilde{S}_{2}$ contained in $\partial S$ such that

$$
x \widetilde{S}_{2} \cap S_{0}^{\prime c l} \neq \phi .
$$

Proof of Theorem 1.3. Let $S$ be a non-closed $K_{\mathbb{C}}-P$ double coset in $G_{\mathbb{C}}$. Then it follows from Lemma 2.3, Lemma 2.4 and Corollary 2.5 that there exist $K_{\mathbb{C}}-B$ double cosets $\widetilde{S}_{1}$ and $\widetilde{S}_{2}$ contained in $\partial S$ such that

$$
x\left(\widetilde{S}_{1} \cup \widetilde{S}_{2}\right) \cap{S_{0}^{\prime}}^{c l} \neq \phi
$$

for all $x \in \partial D$ satisfying

$$
x K_{\mathbb{C}} \in \iota^{-1}\left(\left(G_{\mathbb{R}} c_{\beta_{1}} Q / Q \times G_{\mathbb{R}} \bar{Q} / \bar{Q}\right) \sqcup\left(G_{\mathbb{R}} Q / Q \times G_{\mathbb{R}} \overline{c_{\beta_{1}}} \bar{Q} / \bar{Q}\right)\right) .
$$

Suppose that

$$
y\left(\widetilde{S}_{1} \cup \widetilde{S}_{2}\right)^{c l} \cap S_{0}^{\prime c l}=\phi
$$

for some $y \in \partial D$. Then there exists a neighborhood $U$ of $y$ in $G_{\mathbb{C}}$ such that

$$
x\left(\widetilde{S}_{1} \cup \widetilde{S}_{2}\right)^{c l} \cap S_{0}^{\prime c l}=\phi
$$

for all $x \in U$. But this contradicts (2.5) because the right hand side of (2.6) is dense in $\partial\left(D / K_{\mathbb{C}}\right)$.

\section{3. $S p(2, \mathbb{R})$-CASE}

Let $G_{\mathbb{C}}=S p(2, \mathbb{C})=\left\{g \in G L(4, \mathbb{C}) \mid{ }^{t} g J g=J\right\}$ where

$$
J=\left(\begin{array}{cc}
0 & -I_{2} \\
I_{2} & 0
\end{array}\right) \text {. }
$$

Let

$$
K_{\mathbb{C}}=\left\{\left(\begin{array}{cc}
g & 0 \\
0 & { }^{t} g^{-1}
\end{array}\right) \mid g \in G L(2, \mathbb{C})\right\} \quad \text { and } \quad G_{\mathbb{R}}=G_{\mathbb{C}} \cap U(2,2) \cong S p(2, \mathbb{R}) .
$$


Put $U_{+}=\mathbb{C} e_{1} \oplus \mathbb{C} e_{2}$ and $U_{-}=\mathbb{C} e_{3} \oplus \mathbb{C} e_{4}$ by using the canonical basis $\left\{e_{1}, e_{2}, e_{3}, e_{4}\right\}$ of $\mathbb{C}^{4}$. Then we have

$$
K_{\mathbb{C}}=Q \cap \bar{Q}
$$

where $Q=\left\{g \in G_{\mathbb{C}} \mid g U_{+}=U_{+}\right\}$and $\bar{Q}=\left\{g \in G_{\mathbb{C}} \mid g U_{-}=U_{-}\right\}$.

The full flag manifold $X$ of $G_{\mathbb{C}}$ consists of the flags $\left(V_{1}, V_{2}\right)$ in $\mathbb{C}^{4}$ where $\operatorname{dim} V_{j}=$ $j, V_{1} \subset V_{2}$ and ${ }^{t} u J v=0$ for all $u, v \in V_{2}$. Let $B$ denote the Borel subgroup of $G_{\mathbb{C}}$ defined by

$$
B=\left\{g \in G_{\mathbb{C}} \mid g \mathbb{C} e_{1}=\mathbb{C} e_{1} \text { and } g U_{+}=U_{+}\right\} .
$$

Then the full flag manifold $X$ is identified with $G_{\mathbb{C}} / B$ by the map

$$
g B \mapsto\left(V_{1}, V_{2}\right)=\left(g \mathbb{C} e_{1}, g U_{+}\right) .
$$

There are eleven $K_{\mathbb{C} \text {-orbits }}$

$$
\begin{aligned}
S_{1} & =\left\{\left(V_{1}, V_{2}\right) \mid V_{2}=U_{+}\right\}, \\
S_{2} & =\left\{\left(V_{1}, V_{2}\right) \mid V_{2}=U_{-}\right\}, \\
S_{3} & =\left\{\left(V_{1}, V_{2}\right) \mid V_{1} \subset U_{+}, \operatorname{dim}\left(V_{2} \cap U_{-}\right)=1\right\}, \\
S_{4} & =\left\{\left(V_{1}, V_{2}\right) \mid V_{1} \subset U_{-}, \operatorname{dim}\left(V_{2} \cap U_{+}\right)=1\right\}, \\
S_{5} & =\left\{\left(V_{1}, V_{2}\right) \mid V_{1} \subset U_{+}\right\}-\left(S_{1} \sqcup S_{3}\right), \\
S_{6} & =\left\{\left(V_{1}, V_{2}\right) \mid V_{1} \subset U_{-}\right\}-\left(S_{2} \sqcup S_{4}\right), \\
S_{7} & =\left\{\left(V_{1}, V_{2}\right) \mid \operatorname{dim}\left(V_{2} \cap U_{+}\right)=\operatorname{dim}\left(V_{2} \cap U_{-}\right)=1\right\}-\left(S_{3} \sqcup S_{4}\right), \\
S_{8} & =\left\{\left(V_{1}, V_{2}\right) \mid V_{1} \cap U_{+}=\{0\}, \operatorname{dim}\left(V_{2} \cap U_{+}\right)=1, V_{2} \cap U_{-}=\{0\}\right\}, \\
S_{9} & =\left\{\left(V_{1}, V_{2}\right) \mid V_{1} \cap U_{-}=\{0\}, \operatorname{dim}\left(V_{2} \cap U_{-}\right)=1, V_{2} \cap U_{+}=\{0\}\right\}, \\
S_{10} & =\left\{\left(V_{1}, V_{2}\right) \mid V_{2} \cap U_{ \pm}=\{0\},{ }^{t} v J \tau(v)=0 \text { for } v \in V_{1}\right\}, \\
S_{\text {op }} & =\left\{\left(V_{1}, V_{2}\right) \mid V_{2} \cap U_{ \pm}=\{0\},{ }^{t} v J \tau(v) \neq 0 \text { for } v \in V_{1}-\{0\}\right\}
\end{aligned}
$$

on $X$ where

$$
\tau(v)=\left(\begin{array}{cc}
I_{2} & 0 \\
0 & -I_{2}
\end{array}\right) v
$$

for $v \in \mathbb{C}^{4}$. These orbits are related as follows ([MO, Fig. 12):

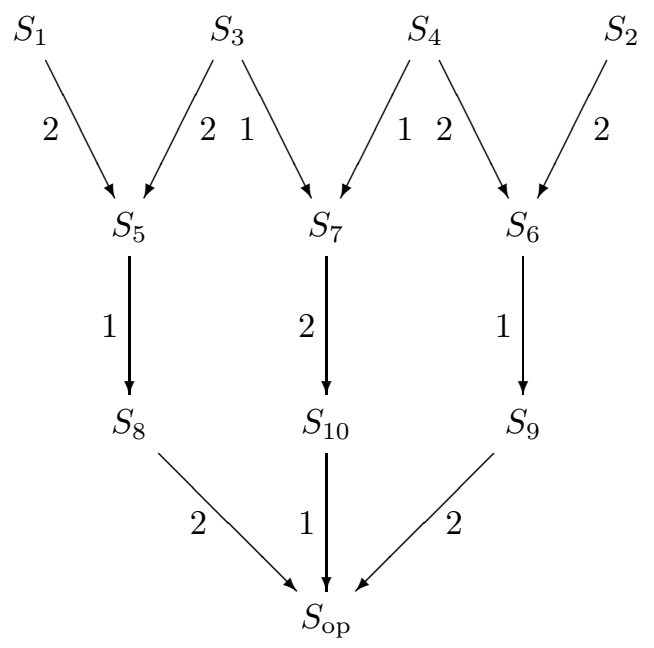


Let $P_{1}$ and $P_{2}$ be the parabolic subgroups of $G_{\mathbb{C}}$ defined by

$$
P_{1}=Q \quad \text { and } \quad P_{2}=\left\{g \in G_{\mathbb{C}} \mid g \mathbb{C} e_{1}=\mathbb{C} e_{1}\right\},
$$

respectively. Then the above diagram implies, for example, that

$$
S_{1} P_{2}=S_{5} P_{2} \quad \text { and that } \quad \operatorname{dim} S_{1}=\operatorname{dim} S_{5}-1
$$

by the arrow attached with the number 2 joining $S_{1}$ and $S_{5}$.

On the other hand define subsets

$$
\begin{gathered}
C_{+}=\left\{z \in \mathbb{C}^{4} \mid(z, z)>0\right\}, \quad C_{-}=\left\{z \in \mathbb{C}^{4} \mid(z, z)<0\right\} \\
\text { and } C_{0}=\left\{z \in \mathbb{C}^{4} \mid(z, z)=0\right\}
\end{gathered}
$$

of $\mathbb{C}^{4}$ using the Hermitian form $(w, z)=\overline{w_{1}} z_{1}+\overline{w_{2}} z_{2}-\overline{w_{3}} z_{3}-\overline{w_{4}} z_{4}$ defining $U(2,2)$. For $v \in \mathbb{C}^{4}$ define subspaces

$$
v^{J}=\left\{u \in \mathbb{C}^{4} \mid{ }^{t} v J u=0\right\} \quad \text { and } \quad v^{\perp}=\left\{u \in \mathbb{C}^{4} \mid(v, u)=0\right\}
$$

of $\mathbb{C}^{4}$. Then $C_{0}$ is devided as $C_{0}=C_{0}^{s} \sqcup C_{0}^{r}$ where

$$
C_{0}^{s}=\left\{v \in C_{0} \mid v^{J}=v^{\perp}\right\} \quad \text { and } \quad C_{0}^{r}=\left\{v \in C_{0} \mid v^{J} \neq v^{\perp}\right\} .
$$

The $G_{\mathbb{R}^{-} \text {-orbits on } X \text { are }}$

$$
\begin{aligned}
S_{1}^{\prime} & =\left\{\left(V_{1}, V_{2}\right) \mid V_{2}-\{0\} \subset C_{+}\right\}, \\
S_{2}^{\prime} & =\left\{\left(V_{1}, V_{2}\right) \mid V_{2}-\{0\} \subset C_{-}\right\}, \\
S_{3}^{\prime} & =\left\{\left(V_{1}, V_{2}\right) \mid V_{1}-\{0\} \subset C_{+}, V_{2} \cap C_{-} \neq \phi\right\}, \\
S_{4}^{\prime} & =\left\{\left(V_{1}, V_{2}\right) \mid V_{1}-\{0\} \subset C_{-}, V_{2} \cap C_{+} \neq \phi\right\}, \\
S_{5}^{\prime} & =\left\{\left(V_{1}, V_{2}\right) \mid V_{1}-\{0\} \subset C_{+}, V_{2} \cap C_{0}^{s} \neq\{0\}\right\}, \\
S_{6}^{\prime} & =\left\{\left(V_{1}, V_{2}\right) \mid V_{1}-\{0\} \subset C_{-}, V_{2} \cap C_{0}^{s} \neq\{0\}\right\}, \\
S_{7}^{\prime} & =\left\{\left(V_{1}, V_{2}\right) \mid V_{1}-\{0\} \subset C_{0}^{r}, V_{2} \not \subset C_{0}\right\}, \\
S_{8}^{\prime} & =\left\{\left(V_{1}, V_{2}\right) \mid V_{1} \subset C_{0}^{s}, V_{2} \cap C_{+} \neq \phi\right\}, \\
S_{9}^{\prime} & =\left\{\left(V_{1}, V_{2}\right) \mid V_{1} \subset C_{0}^{s}, V_{2} \cap C_{-} \neq \phi\right\}, \\
S_{10}^{\prime} & =\left\{\left(V_{1}, V_{2}\right) \mid V_{1}-\{0\} \subset C_{0}^{r}, V_{2} \subset C_{0}\right\}, \\
S_{\text {op }}^{\prime} & =\left\{\left(V_{1}, V_{2}\right) \mid V_{1} \subset C_{0}^{s}, V_{2} \subset C_{0}\right\} .
\end{aligned}
$$

Here the $K_{\mathbb{C}^{-o r b i t}} S_{j}$ and the $G_{\mathbb{R} \text {-orbit }} S_{j}^{\prime}$ correspond by the duality for each $j=1, \ldots, 10$, op.

Take a maximal abelian subspace

$$
\mathfrak{j}=\left\{Y\left(a_{1}, a_{2}\right)=\left(\begin{array}{cccc}
a_{1} & 0 & 0 & 0 \\
0 & a_{2} & 0 & 0 \\
0 & 0 & -a_{1} & 0 \\
0 & 0 & 0 & -a_{2}
\end{array}\right) \mid a_{1}, a_{2} \in \mathbb{R}\right\}
$$

of $i \mathfrak{m}$. Using the linear forms $e_{j}: Y\left(a_{1}, a_{2}\right) \mapsto a_{j}$ for $j=1,2$, we can write

$$
\Delta=\left\{ \pm 2 e_{1}, \pm 2 e_{2}, \pm e_{1} \pm e_{2}\right\} \quad \text { and } \quad \Delta_{n}^{+}=\left\{2 e_{1}, 2 e_{2}, e_{1}+e_{2}\right\} .
$$

Write $\beta_{1}=2 e_{1}, \beta_{2}=2 e_{2}$ and $\delta=e_{1}+e_{2}$. Take root vectors $X_{1}=-E_{13}$ of $\mathfrak{g}_{\mathbb{C}}\left(\mathfrak{j}, \beta_{1}\right)$ and $X_{2}=-E_{24}$ of $\mathfrak{g}_{\mathbb{C}}\left(\mathfrak{j}, \beta_{2}\right)$ where $E_{i j}(i, j=1, \ldots, 4)$ denotes the matrix units. 
Define

$$
t_{1}(s)=\exp s\left(X_{1}-\overline{X_{1}}\right)=\exp s\left(E_{31}-E_{13}\right)=\left(\begin{array}{cccc}
\cos s & 0 & -\sin s & 0 \\
0 & 1 & 0 & 0 \\
\sin s & 0 & \cos s & 0 \\
0 & 0 & 0 & 1
\end{array}\right)
$$

and

$$
t_{2}(s)=\exp s\left(X_{2}-\overline{X_{2}}\right)=\exp s\left(E_{42}-E_{24}\right)=\left(\begin{array}{cccc}
1 & 0 & 0 & 0 \\
0 & \cos s & 0 & -\sin s \\
0 & 0 & 1 & 0 \\
0 & \sin s & 0 & \cos s
\end{array}\right)
$$

for $s \in \mathbb{R}$. Then we can write the Akhiezer-Gindikin domain $D$ as

$$
D=G_{\mathbb{R}} T^{+} K_{\mathbb{C}}
$$

where $T^{+}=\left\{t_{1}\left(s_{1}\right) t_{2}\left(s_{2}\right)|| s_{1}|<\pi / 4,| s_{2} \mid<\pi / 4\right\}$. Write $c_{\beta_{j}}=t_{j}(\pi / 4)$ and $w_{\beta_{j}}=t_{j}(\pi / 2)$ for $j=1,2$. Then we can write

$$
S_{j}=K_{\mathbb{C}} g B \quad \text { and } \quad S_{j}^{\prime}=G_{\mathbb{R}} g B
$$

for $j=1, \ldots, 10$, op with the following representatives $g$ ([M1], Theorem 2):

\begin{tabular}{|c|c|c|c|c|c|c|c|c|c|c|c|}
\hline$j$ & 1 & 2 & 3 & 4 & 5 & 6 & 7 & 8 & 9 & 10 & op \\
\hline$g$ & $e$ & $w_{\beta_{1}} w_{\beta_{2}}$ & $w_{\beta_{2}}$ & $w_{\beta_{1}}$ & $c_{\beta_{2}}$ & $c_{\beta_{2}} w_{\beta_{1}}$ & $c_{\delta} w_{\beta_{2}}$ & $c_{\beta_{1}}$ & $c_{\beta_{1}} w_{\beta_{2}}$ & $c_{\delta}$ & $c_{\beta_{1}} c_{\beta_{2}}$ \\
\hline
\end{tabular}

Here

$$
c_{\delta}=\frac{1}{\sqrt{2}}\left(\begin{array}{cccc}
1 & 0 & 0 & -1 \\
0 & 1 & -1 & 0 \\
0 & 1 & 1 & 0 \\
1 & 0 & 0 & 1
\end{array}\right)=\exp \frac{\pi}{4}\left(X_{\delta}-\overline{X_{\delta}}\right)
$$

with $X_{\delta}=-\left(E_{14}+E_{23}\right) \in \mathfrak{g}_{\mathbb{C}}(\mathfrak{j}, \delta)$.

The standard maximal flag manifold $G_{\mathbb{C}} / Q$ is identified with the space $Y$ of two dimensional subspaces $V_{+}$of $\mathbb{C}^{4}$ such that ${ }^{t} u J v=0$ for all $u, v \in V_{+}$by the map

$$
G_{\mathbb{C}} / Q \ni g Q \mapsto V_{+}=g U_{+} \in Y .
$$

Similarly we also identify $G_{\mathbb{C}} / \bar{Q}$ with $Y$ by the map

$$
G_{\mathbb{C}} / \bar{Q} \ni g \bar{Q} \mapsto V_{-}=g U_{-} \in Y .
$$

As in Section 2 the complex symmetric space $G_{\mathbb{C}} / K_{\mathbb{C}}$ is naturally identified with the open subset

$$
\left\{\left(V_{+}, V_{-}\right) \in G_{\mathbb{C}} / Q \times G_{\mathbb{C}} / \bar{Q} \mid V_{+} \cap V_{-}=\{0\}\right\}
$$

of $G_{\mathbb{C}} / Q \times G_{\mathbb{C}} / \bar{Q} \cong Y \times Y$ by the map

$$
\iota: g K_{\mathbb{C}} \mapsto\left(V_{+}, V_{-}\right)=\left(g U_{+}, g U_{-}\right) .
$$

Then the Akhiezer-Gindikin domain $D / K_{\mathbb{C}}$ is identified with

$$
G_{\mathbb{R}} Q / Q \times G_{\mathbb{R}} \bar{Q} / \bar{Q}=\left\{\left(V_{+}, V_{-}\right) \in Y \times Y \mid V_{+}-\{0\} \subset C_{+} \text {and } V_{-}-\{0\} \subset C_{-}\right\} .
$$

Let $x K_{\mathbb{C}}$ be an element of $\partial\left(D / K_{\mathbb{C}}\right)$ such that $\iota\left(x K_{\mathbb{C}}\right) \in G_{\mathbb{R}} c_{\beta_{1}} Q / Q \times G_{\mathbb{R}} \bar{Q} / \bar{Q}$. Then it follows from Lemma 2.3 that

$$
x K_{\mathbb{C}} g B \cap G_{\mathbb{R}} c_{\beta_{1}} g B \neq \phi
$$


for $g=e, w_{\beta_{2}}$ and $c_{\beta_{2}}$. This implies that

$$
\begin{aligned}
& x S_{1} \cap S_{8}^{\prime} \neq \phi, \\
& x S_{3} \cap S_{9}^{\prime} \neq \phi
\end{aligned}
$$

and that

$$
x S_{5} \cap S_{\text {op }}^{\prime} \neq \phi .
$$

Since $S_{7}^{\prime c l}=\left\{\left(V_{1}, V_{2}\right) \mid V_{1} \subset C_{0}\right\} \supset S_{9}^{\prime}$, it follows from (3.2) that

$$
x S_{3} \cap S_{7}^{\prime c l} \neq \phi .
$$

On the other hand since $S_{10}^{\prime c l} \supset S_{\mathrm{op}}^{\prime}$, it follows from (3.3) that

$$
x S_{5} \cap S_{10}^{\prime c l} \neq \phi .
$$

Remark 3.1. (i) If $\iota\left(x K_{\mathbb{C}}\right) \in G_{\mathbb{R}} Q / Q \times G_{\mathbb{R}} \overline{c_{\beta_{1}}} \bar{Q} / \bar{Q}$, then we can prove

$$
\begin{gathered}
x S_{2} \cap S_{9}^{\prime} \neq \phi, \quad x S_{4} \cap S_{8}^{\prime} \neq \phi, \quad x S_{6} \cap S_{\mathrm{op}}^{\prime} \neq \phi, \\
x S_{4} \cap S_{7}^{\prime c l} \neq \phi \quad \text { and } \quad x S_{6} \cap S_{10}^{\prime c l} \neq \phi
\end{gathered}
$$

in the same way.

(ii) If we apply M4, Theorem 1.3, to this case, then we have

$$
x \in \partial D \Longrightarrow x\left(S_{5} \sqcup S_{6}\right)^{c l} \cap S_{\text {op }}^{\prime} \neq \phi .
$$

So we see that the results in this paper are refinements of this theorem for Hermitian cases.

By (3.4) and (3.5) we proved the following.

Proposition 3.2. If $\iota\left(x K_{\mathbb{C}}\right) \in G_{\mathbb{R}} c_{\beta_{1}} Q / Q \times G_{\mathbb{R}} \bar{Q} / \bar{Q}$, then we have:

(i) $x K_{\mathbb{C}} w_{\beta_{2}} B \cap\left(G_{\mathbb{R}} c_{\delta} w_{\beta_{2}} B\right)^{c l} \neq \phi$.

(ii) $x K_{\mathbb{C}} c_{\beta_{2}} B \cap\left(G_{\mathbb{R}} c_{\delta} B\right)^{c l} \neq \phi$.

Remark 3.3. It is clear that $K_{\mathbb{C}} w_{\beta_{2}} B=S_{3} \subset S_{7}^{c l}=\left(K_{\mathbb{C}} c_{\delta} w_{\beta_{2}} B\right)^{c l}$ and that $K_{\mathbb{C}} c_{\beta_{2}} B=S_{5} \subset S_{10}^{c l}=\left(K_{\mathbb{C}} c_{\delta} B\right)^{c l}$.

\section{ACKNOWLEDGEMENT}

The author would like to express his hearty thanks to S. Gindikin for valuable suggestions and encouragements.

\section{REFERENCES}

[AG] D. N. Akhiezer and S. G. Gindikin, On Stein extensions of real symmetric spaces, Math. Ann. 286 (1990), 1-12. MR.1032920 (91a:32047)

[B] L. Barchini, Stein extensions of real symmetric spaces and the geometry of the flag manifold, Math. Ann. 326 (2003), 331-346. MR1990913 (2004d:22007)

[BHH] D. Burns, S. Halverscheid and R. Hind, The geometry of Grauert tubes and complexification of symmetric spaces, Duke Math. J. 118 (2003), 465-491. MR1983038 (2004g:32025)

$[\mathrm{FH}]$ G. Fels and A. Huckleberry, Characterization of cycle domains via Kobayashi hyperbolicity, Bull. Soc. Math. France 133 (2005), 121-144. MR2145022

[GM1] S. Gindikin and T. Matsuki, Stein extensions of Riemannian symmetric spaces and dualities of orbits on flag manifolds, Transform. Groups 8 (2003), 333-376. MR2015255 (2005b:22017)

[GM2] S. Gindikin and T. Matsuki, A remark on Schubert cells and the duality of orbits on flag manifolds, J. Math. Soc. Japan 57 (2005), 157-165. MR2114726 (2005j:14070) 
[H] A. Huckleberry, On certain domains in cycle spaces of flag manifolds, Math. Ann. 323 (2002), 797-810. MR1924279 (2003g:32037)

[M1] T. Matsuki, The orbits of affine symmetric spaces under the action of minimal parabolic subgroups, J. Math. Soc. Japan 31 (1979), 331-357. MR0527548 (81a:53049)

[M2] T. Matsuki, Closure relations for orbits on affine symmetric spaces under the action of parabolic subgroups. Intersections of associated orbits, Hiroshima Math. J. 18 (1988), 59-67. MR0935882 (89f:53073)

[M3] T. Matsuki, Stein extensions of Riemann symmetric spaces and some generalization, J. Lie Theory 13 (2003), 563-570. MR2003160 (2004i:53062)

[M4] T. Matsuki, Equivalence of domains arising from duality of orbits on flag manifolds, Trans. Amer. Math. Soc. 358 (2006), 2217-2245. MR2197441

[M5] T. Matsuki, Equivalence of domains arising from duality of orbits on flag manifolds II, Proc. Amer. Math. Soc. 134 (2006), 3423-3428. MR.2240651

[MO] T. Matsuki and T. Oshima, Embeddings of discrete series into principal series. In The Orbit Method in Representation Theory, Birkhäuser, 1990, 147-175. MR 1095345 (92d:22020)

[Sp] T. A. Springer, Some results on algebraic groups with involutions, Adv. Stud. Pure Math. 6 (1984), 525-534. MR0803346 (86m:20050)

[WW] R. O. Wells and J. A. Wolf, Poincaré series and automorphic cohomology on flag domains, Annals of Math. 105 (1977), 397-448. MR0447645 (56:5955)

[WZ1] J. A. Wolf and R. Zierau, Cayley transforms and orbit structure in complex flag manifolds, Transform. Groups 2 (1997), 391-405. MR.1486038 (99b:32049)

[WZ2] J. A. Wolf and R. Zierau, Linear cycle spaces in flag domains, Math. Ann. 316 (2000), 529-545. MR 1752783 (2001g:32054)

[WZ3] J. A. Wolf and R. Zierau, A note on the linear cycle spaces for groups of Hermitian type, J. Lie Theory 13 (2003), 189-191. MR1958581 (2004a:22015)

Department of Mathematics, Faculty of Science, Kyoto University, Kyoto 606-8502, JAPAN

E-mail address: matsuki@math.kyoto-u.ac.jp 